\title{
Percutaneous closure of a large atrial septal defect in a child with severe dextroscoliosis: A case report
}

\author{
ADRIAN LINTE $^{1}$, ANDRA RODICA BALANESCU ${ }^{2}$, EUSTAQUIO ONORATO $^{3}$, \\ DINU VALENTIN BALANESCU ${ }^{2}$, VIOLETA CLAUDIA BOJINCA ${ }^{2}$, ELENA DRAKONAKI ${ }^{4}$, \\ MIHAI BOJINCA ${ }^{5}$ and SERBAN MIHAI BALANESCU ${ }^{1}$
}

\begin{abstract}
${ }^{1}$ Department of Cardiology, Elias Emergency University Hospital, 'Carol Davila' University of Medicine and Pharmacy, 011461 Bucharest; ${ }^{2}$ Department of Internal Medicine and Rheumatology, 'Sfanta Maria' Hospital, 'Carol Davila' University of Medicine and Pharmacy, 011172 Bucharest, Romania; ${ }^{3}$ Department of Cardiovascular, Humanitas Gavazzeni Clinic, I-24125 Bergamo, Italy; ${ }^{4}$ Independent Radiology Practice, 71202 Heraklion, Greece;

${ }^{5}$ Department of Internal Medicine and Rheumatology, 'Dr. I. Cantacuzino' Clinical Hospital, 'Carol Davila' University of Medicine and Pharmacy, 030167 Bucharest, Romania
\end{abstract}

Received August 30, 2018; Accepted October 10, 2018

DOI: 10.3892/etm.2018.6991

\begin{abstract}
In this study, we present the case of an 11-yearold child with cachexia, severe dextroscoliosis, pectus carinatum, secondary restrictive pulmonary disease, and an incidental finding of a large ostium secundum atrial septal defect (ASD) that was identified on preoperative assessment for surgical correction of the spinal deformity. Transthoracic and transesophageal echocardiography confirmed significant left-to-right shunting with a pulmonary blood flow to systemic blood flow ratio (Qp/Qs) of 3.18 through a $14 \mathrm{~mm}$ defect with good circumferential rims, larger than $5 \mathrm{~mm}$. Despite significant left-to-right shunting, the defect was found prior to the development of pulmonary hypertension or right heart enlargement. The decision to close the atrial septal defect by percutaneous intervention was preferred over surgical closure, due to the restrictive pulmonary physiology and low body mass index $\left(12.6 \mathrm{~kg} / \mathrm{m}^{2}\right)$. Percutaneous closure of the ASD was successfully performed under general anesthesia using an Occlutech Figulla Flex II ASD device, with no residual shunt. Surgical correction of the dextroscoliosis was subsequently performed with good results. A comprehensive clinical and
\end{abstract}

Correspondence to: Dr Andra Rodica Balanescu, Department of Internal Medicine and Rheumatology, 'Sfanta Maria' Hospital, 'Carol Davila' University of Medicine and Pharmacy, 37-39 Ion Mihalache Bd., 011172 Bucharest, Romania

E-mail: balanescu.andra@gmail.com

Abbreviations: ASD, atrial septal defect; CHD, congenital heart disease; Qp/Qs, pulmonary blood flow to systemic blood flow ratio; TEE, transesophageal echocardiogram; TTE, transthoracic echocardiogram

Key words: atrial septal defect, endovascular treatment, scoliosis echocardiographic evaluation is needed in patients with skeletal abnormalities in order to rule out associated congenital heart defects, which may impose therapeutic challenges.

\section{Introduction}

Congenital skeletal abnormalities are common conditions that can generally be surgically corrected in the first few months of life or during childhood (1). The incidence of congenital cardiac abnormalities is increased in patients with congenital scoliosis compared to the general population $(1,2)$. Therefore, the presence of congenital scoliosis imposes a detailed echocardiographic assessment $(1,2)$. Atrial septal defects (ASDs) account for $7-10 \%$ of all congenital heart diseases (CHDs) in children and is found in $13 \%$ of patients with congenital scoliosis $(3,4)$. If untreated, ASDs are associated with a significant risk for atrial arrhythmias and cardiovascular morbidity. Closure of hemodynamically significant defects is, thus, advised (3). Although surgery continues to be the gold standard therapy, percutaneous ASD closure has increased in use and has comparable outcomes $(3,4)$.

We present the case of an 11-year-old girl with a complex chest wall deformity who was scheduled for surgical correction of severe congenital scoliosis. Preoperative clinical and echocardiographic evaluation showed the presence of a large ostium secundum ASD with a significant left-to-right shunt which required closure prior to orthopedic surgery for the skeletal disease. Percutaneous implantation of a dedicated device was preferred over surgical intervention due to the chest deformity and the pulmonary restrictive disease.

\section{Case report}

An 11-year-old girl with complex thoracic skeletal deformity, congenital scoliosis, and pectus carinatum (Fig. 1A) was referred for cardiovascular assessment prior to surgical correction of the spinal defect. Her weight was $15 \mathrm{~kg}$ and her 

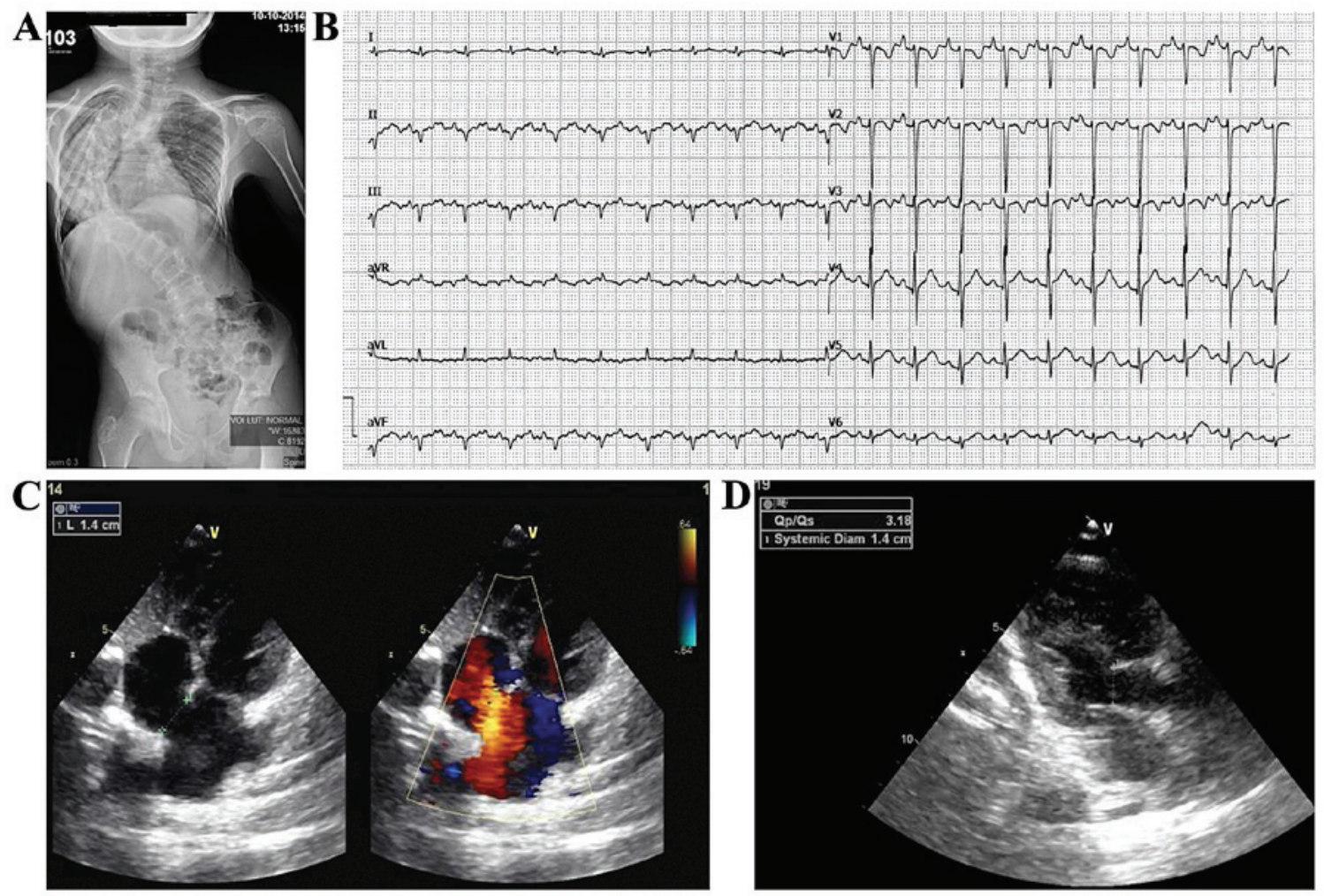

Figure 1. An 11-year-old girl with severe dextroscoliosis and ostium secundum atrial septal defect. (A) Severe dextroscoliosis on spinal X-ray (pasting technique). (B) Electrocardiogram showing sinus rhythm with P pulmonale, left axis deviation, S waves with high amplitude in the left precordial leads (C) Transthoracic echocardiography (4 chamber subcostal view): Ostium secundum type atrial septal defect with a diameter of $14 \mathrm{~mm}$ and a left-to-right shunt.

(D) Transthoracic echocardiography: pulmonary to systemic flow ratio (Qp/Qs) was 3.18 .

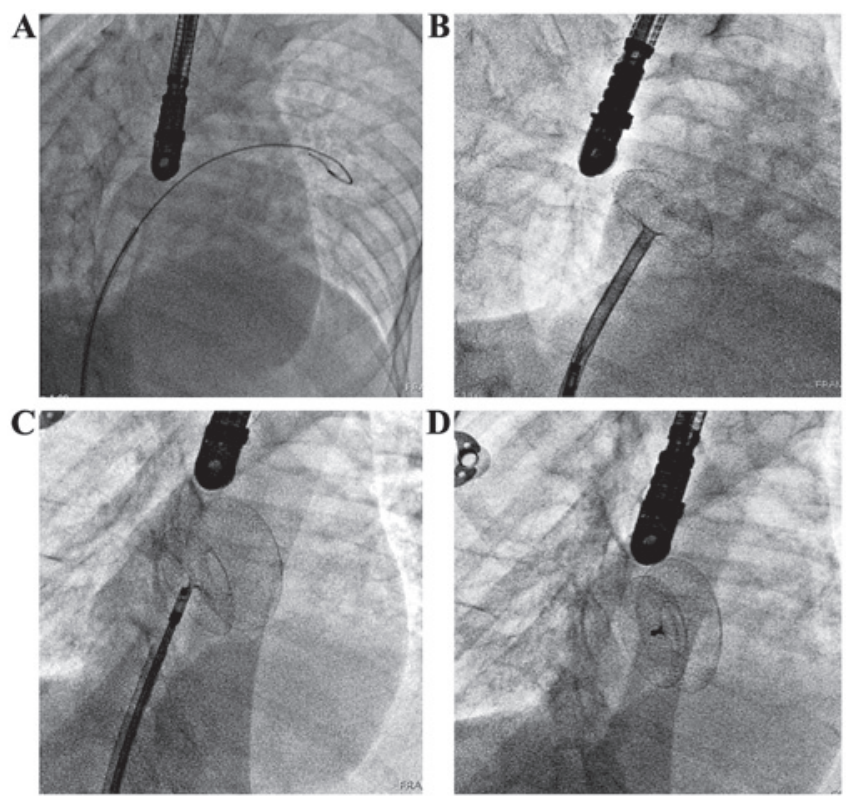

Figure 2. Endovascular closure of an ostium secundum atrial septal defect in an 11-year-old girl with severe dextroscoliosis. (A) Angiography in $30^{\circ}$ left anterior oblique view: The stiff guidewire is placed in the superior left pulmonary vein. (B) Angiography in $30^{\circ}$ left anterior oblique view: Release of the left disk of the device. (C) Angiography in anteroposterior view: Release of the right disk of the device. (D) Angiography in anteroposterior view: Final position of the Occlutech Figulla Flex II ASD device.

height was $109 \mathrm{~cm}$, with a body mass index of $12.6 \mathrm{~kg} / \mathrm{m}^{2}$. Cardiac examination revealed fixed splitting of the 2 nd heart sound, no heart murmurs, a blood pressure of 120/70 mmHg, and a heart rate of 70 beats $/ \mathrm{min}$. Physical exam was otherwise unremarkable, with the exception of the spinal deformity. Spirometry revealed severe restrictive pulmonary dysfunction with a vital capacity at $26 \%$ of predicted value. Routine blood tests were normal, with normal kidney and liver function.

An electrocardiogram showed sinus rhythm with 'pulmonary' $\mathrm{P}$ waves and left axis deviation (due to intrathoracic cardiac orientation) (Fig. 1B). A transthoracic echocardiogram (TTE) revealed a 14-mm ostium secundum ASD, left-to-right shunting (Fig. 1C), and a ratio of pulmonary blood flow to systemic blood flow (Qp/Qs) of 3.18 (Fig. 1D). The right cardiac chambers were mildly dilated. Estimated pulmonary artery pressure was normal $(25 \mathrm{mmHg})$. There were no structural changes of the left cardiac chambers or valves and left ventricular ejection fraction was normal. A transesophageal echocardiogram (TEE) was performed; ASD diameter was measured at $14 \mathrm{~mm}$ and septal length at $34 \mathrm{~mm}$. The defect was located in the central atrial septum and all the muscular rims were $>5 \mathrm{~mm}$.

Percutaneous treatment of the ASD was the preferred treatment approach, given the high surgical risk in a cachectic patient with severe restrictive pulmonary dysfunction. All the criteria for percutaneous ASD closure were met (3). The Figulla Flex II (Occlutech GmbH, Jena, Germany) device was chosen, due to its good flexibility and our previous experience. A $16.5 \mathrm{~mm}$ device was considered appropriate based on TEE assessment. 
The intervention was performed in the catheterization laboratory under general anesthesia with orotracheal intubation and mechanical ventilation. The procedure was guided by multiplane transesophageal echocardiography. The right femoral vein was punctured and a $6 \mathrm{~F}$ sheath was inserted. A 6F MP1 catheter was advanced via a 0.035 inch, $180 \mathrm{~cm}$ long guidewire through the atrial septal defect into the left atrium and then further into the left upper pulmonary vein. The standard antero-posterior angiographic view was modified in order to adjust for the intrathoracic cardiac orientation secondary to the chest wall anomaly; therefore, the proper working projection was left anterior oblique $30^{\circ}$ (Fig. 2A). The 0.035-inch guidewire was exchanged for a 0.035 inch $260 \mathrm{~cm}$ long Occlutech stiff guidewire (Fig. 2A) which allowed for the exchange of the $6 \mathrm{~F}$ sheath with a long dedicated introducer-sheath with the tip placed in the left atrium. The $16.5 \mathrm{~mm}$ Figulla Flex II device was advanced through the introducer sheath and the left disk was released into the left atrium after gentle clockwise rotation of the introducer-sheath. The introducer sheath and the device were pulled against the atrial septum until the apposition of the left disc on the septum was obtained and then the right disc was released into the right atrium (Fig. 2B and 2C). After verifying for good stability and apposition, the device was released (Fig. 2D) with good final procedural results. Complete exclusion of the ASD and no residual shunting on TEE. The patient was transferred to the postoperative intensive care unit where she was extubated. She was soon transferred to the inpatient unit where she was kept under surveillance for 2 more days. TTE at discharge showed no residual shunt. The patient was treated with aspirin $75 \mathrm{mg}$ once daily for 6 months following the procedure. Surgical correction of the spinal deformity was performed 6 months after the procedure without complications. The patient was in good health at 2.5 year follow-up.

\section{Discussion}

Our case report describes the association of a major skeletal birth defect (congenital dextroscoliosis and pectus carinatum) with one of the most frequent congenital heart diseases (ostium secundum type ASD). Craniofacial and skeletal abnormalities are often associated with congenital heart disease $(1,2)$. This observation has important clinical consequences, as these patients often require extensive evaluation and carefully selected treatment strategies.

Although the clinical association between kyphoscoliosis and congenital heart defects is well recognized $(1,2)$, few large-scale epidemiological studies have addressed this issue. In adolescent idiopathic scoliosis, the prevalence of structural cardiac abnormalities ranges between 3.6-17.5\% (5,6). A retrospective cohort of mixed congenital spinal deformities reports $14.1-26 \%$ prevalence of structural cardiac abnormalities $(7,8)$ in patients with congenital scoliosis. The authors also report that congenital kyphosis is more frequently associated with heart defects. The prevalence of ASD in patients with congenital scoliosis ranges between $2.5 \%$ (7) and $13 \%$ (4); a prevalence of $8.75 \%$ is reported in a study addressing idiopathic scoliosis (6). As a consequence, some experts recommend routine echocardiographic screening for congenital heart disease in patients with congenital kyphoscoliosis $(1,2,5,8)$.

To the best of our knowledge, there are only a few articles in the literature presenting cases of percutaneous ASD closure in patients with severe congenital scoliosis $(9,10)$. The chest anatomy of patients with scoliosis imposes technical difficulties with performing endovascular procedures. The position of the $\mathrm{C}$-arm needs to be adapted to compensate for the intrathoracic cardiac orientation. A $30^{\circ}$ left anterior oblique projection was chosen to advance the guidewire in the left superior pulmonary vein and for the proper positioning of the long introducer sheath. The introducer sheath needed to be turned clockwise in order to obtain a good alignment and to minimize the tension in the device during its release. This ensured that the device was properly positioned with a minimum risk of slippage of the left disk into the right atrium during release. After verifying for correct positioning with TEE and stability check with push-pull maneuver, the device was safely released and TEE demonstrated complete closure of the atrial septal device with no residual shunt.

A comprehensive clinical and echocardiographic evaluation is needed in patients with skeletal abnormalities in order to screen for associated congenital heart defects. The current treatment of choice for most ASDs is percutaneous closure with dedicated devices. In the case of simultaneous ASD and kyphoscoliosis, special consideration should be given to the intrathoracic cardiac orientation. A modified cath lab protocol is imposed, including adaptation of the angiographic working projection, the correct alignment of the long introducer-sheath, and the release time of the occluder device. Percutaneous techniques can be successfully performed in patients with ASD and secondary restrictive pulmonary disease, despite challenging anatomy due to severe chest wall deformity. This approach should, therefore, be preferred over surgical closure.

\section{Acknowledgements}

The authors would like to acknowledge Antonela Muresan, Department of Anesthesiology, Cardiopediatric Center, Monza Hospital (Bucharest, Romania), and Dana Constantinescu, Department of Cardiology, Monza Hospital (Bucharest, Romania), who were part of the team treating and caring for this patient and contributed to data collection.

\section{Funding}

No funding was received.

\section{Availability of data and materials}

Not applicable.

\section{Authors' contributions}

AL, ARB, EO, DVB and SMB contributed to drafting and writing the manuscript. AL, EO and SMB were involved in the care of patient. ARB and DVB contributed to the organization, analysis and interpretation of data. VCB, ED, and MB were involved in the critical revision of the manuscript, the 
organization of data, the gathering and the image formation. SMB coordinated the group and the preparation of this manuscript. All authors read and approved the final version of the manuscript.

\section{Ethics approval and consent to participate}

The need for Ethics approval was waived as this is a report of clinical practice and does not constitute biomedical research. Reporting is consistent with all ethical requirements.

\section{Patient consent for publication}

Informed consent was obtained from the patient's family. All identifying information was removed from this manuscript.

\section{Competing interests}

The authors declare that they have no competing interests.

\section{References}

1. Tikoo A, Kothari MK, Shah K and Nene A: Current concepts congenital scoliosis. Open Orthop J 11: 337-345, 2017.
2. Bozcali E, Ucpunar H, Sevencan A, Balioglu MB, Albayrak A and Polat V: A retrospective study of congenital cardiac abnormality associated with scoliosis. Asian Spine J 10: 226-230, 2016.

3. Kazmouz S, Kenny D, Cao QL, Kavinsky CJ and Hijazi ZM: Transcatheter closure of secundum atrial septal defects. J Invasive Cardiol 25: 257-264, 2013.

4. Vasquez AF and Lasala JM: Atrial septal defect closure. Cardiol Clin 31: 385-400, 2013.

5. Ipp L, Flynn P, Blanco J, Green D, Boachie-Adjei O, Kozich J, Chan G, Denneen J and Widmann R: The findings of preoperative cardiac screening studies in adolescent idiopathic scoliosis. J Pediatr Orthop 31: 764-766, 2011.

6. Liu L, Xiu P, Li Q, Song Y, Chen R and Zhou C: Prevalence of cardiac dysfunction and abnormalities in patients with adolescent idiopathic scoliosis requiring surgery. Orthopedics 33: 882, 2010.

7. Liu YT, Guo LL, Tian Z, Zhu WL, Yu B, Zhang SY and Qiu GX: A retrospective study of congenital scoliosis and associated cardiac and intraspinal abnormities in a Chinese population. Eur Spine J 20: 2111-2114, 2011

8. Basu PS, Elsebaie H and Noordeen MH: Congenital spinal deformity: A comprehensive assessment at presentation. Spine 27: 2255-2259, 2002.

9. Lanjewar CP, Khothari S and Kerkar PG: Delivery sheath tear without modification during ASD closure in a patient with kyphoscoliosis. Catheter Cardiovasc Interv 69: 766-767, 2007.

10. Mangovski L, Farkić M and Jovović L: Transcatheter closure of atrial septal defect in a patient with Noonan syndrome after corrective surgery. Vojnosanit Pregl 72: 557-560, 2015. 